Die Regulierung der klinischen Forschung in der Europäischen Union

Dagron, Stéphanie

Posted at the Zurich Open Repository and Archive, University of Zurich ZORA URL: https://doi.org/10.5167/uzh-104383

Book Section

Originally published at:

Dagron, Stéphanie (2014). Die Regulierung der klinischen Forschung in der Europäischen Union. In: Lenk, Christian; Duttge, Gunnar; Gangerau, Heiner. Handbuch Ethik und Recht der Forschung am Menschen. Heidelberg: Springer, 525-530. 


\title{
Die Regulierung der klinischen Forschung in der Europäischen Union
}

\author{
Stéphanie Dagron
}

\section{Einleitung}

Die europäische Richtlinie 2001/20/EG vom 4. April 2001 zur Angleichung der Rechts- und Verwaltungsvorschriften der Mitgliedstaaten über die Anwendung der guten klinischen Praxis (GCP-RL) ist das erste für alle Mitgliedstaaten der europäischen Union in diesem Bereich verbindliche Instrument. Sie enthält die Bedingungen für die Beantragung, Durchführung, Aufzeichnung, Berichterstattung, Analyse der Ergebnisse und Überwachung der klinischen Prüfungen, die im Zusammenhang mit dem Zulassungsverfahren von Arzneimitteln geführt werden müssen. Diese Richtlinie wurde 2005 durch die Richtlinie 2005/28/EG vom 8. April 2005 (2005RL) ergänzt, die ausführliche Bestimmungen über die Durchführung der Prüfung an sich beinhaltet.

Durch die Angleichung der nationalen Bestimmungen für die Durchführung der klinischen Prüfungen verfolgen diese Richtlinien zwei wichtige Ziele. Erstens soll der Schutz der Rechte, der Sicherheit und des Wohlergehens der an diesen klinischen Prüfungen teilnehmenden Personen durch die Beachtung ethischer und wissenschaftlicher Anforderungen in allen Mitgliedstaaten gewährleistet werden. Zweitens sollen der europäische Arzneimittelbinnenmarkt gestärkt und die Industrie gefördert werden. Die Balance zwischen diesen Zielen ist schwer zu erreichen, da die Anforderungen für den Probandenschutz oft zusätzliche Verpflichtungen darstellen, die für die Pharmaindustrie sowie für andere Sponsoren einer klinischen Prüfung einen hohen administrativen Aufwand bedeuten können (Sewing 2011). Die europäische Kommission hat im Juni 2012 einen Vorschlag für eine Verordnung über klinische Prüfungen und zur Aufhebung der beiden Richtlinien veröffentlicht, die diese Balance neu definieren soll.

\footnotetext{
S. Dagron $(\bowtie)$

Rechtswissenschaftliches Institut, Universität Zürich,

Treichlerstrasse 10, 8032 Zürich, Schweiz

E-Mail: stephanie.dagron@rwi.uzh.ch

C. Lenk et al. (Hrsg.), Handbuch Ethik und Recht der Forschung am Menschen, DOI 10.1007/978-3-642-35099-3 83, (c) Springer-Verlag Berlin Heidelberg 2014
} 


\section{Der Schutz der Probanden}

Der Schutz der Menschenrechte und der Würde des Menschen steht im Zentrum der europäischen Richtlinien zur guten klinischen Praxis, wie in den Erwägungsgründen ausgeführt wird. Der Schutz wird durch verschiedene Mechanismen gewährleistet, mit denen ,die Rechte, die Sicherheit und das Wohlergehen der Teilnehmer an klinischen Prüfungen geschützt werden“" (Art. 1 (2), GCP-RL; Art. 2, 2005-RL).

Eine zentrale Rolle spielen die Ethikkommissionen, die in allen Mitgliedstaaten einzurichten sind. Besonders wichtig ist die Rolle der Ethikkommission vor dem Beginn der klinischen Prüfung. Gemäß Art. 9 der GCP-Richtlinie darf eine klinische Prüfung, die kein positives Votum der Ethikkommission zur wissenschaftlichen und ethischen Durchführbarkeit des Prüfplans bekommen hat, nicht durchgeführt werden. In ihrer Stellungnahme soll die Ethikkommission folgende Elemente prüfen, die den Schutz der Probanden sichern sollen: Die Relevanz der klinischen Prüfung und ihrer Planung, die Risiken und der erwartete Nutzen, die Qualität der Einrichtung, die Qualifikation des Prüfers und seiner Mitarbeiter, die Durchführung der Aufklärung, das Vorhandensein einer Versicherung und die Modalitäten für die Auswahl der Prüfungsteilnehmer (Art. 6, GCP-RL).

Einen wesentlichen Mechanismus zum Schutz der Probanden stellt die schriftliche Einwilligung des Prüfungsteilnehmers dar, die aufgrund angemessener und vollständiger Information zu den Zielen, Risiken, Nachteilen und Bedingungen der Durchführung sowie über das Recht, seine Teilnahme jederzeit zu beenden, abgegeben werden muss (Art. 3 (b), 6 (g), GCP-RL). Die Bedingungen der Teilnahme von Personen, die nicht rechtswirksam in eine klinische Prüfung einwilligen können (mit der Ausnahme der Notsituation, die nicht in der GCP-RL geregelt ist), sind besonders präzise definiert (Erwägungsgrund 3, Art. 4 und Art. 5, GCP-RL). Minderjährige und einwilligungsunfähige Erwachsene dürfen nur unter besonderen Bedingungen in klinische Studien einbezogen werden. Das bedeutet für nicht einwilligungsfähige Erwachsene insbesondere, dass sie nur in Versuche eingeschlossen werden dürfen, die einen unmittelbaren Nutzen für sie haben. Bei Minderjährigen darf dagegen eine klinische Prüfung nicht nur durchgeführt werden, wenn die Forschung sich unmittelbar auf den klinischen Zustand des Patienten bezieht, sondern auch wenn die Forschung ihrem Wesen nach nur an Minderjährigen durchgeführt werden kann (Art. 4e, GCP-RL). Weitere Bedingungen betreffen unmittelbar die Art der Prüfungen, die „mit möglich wenig Schmerzen, Beschwerden, Angst und anderen vorhersehbaren Risiken verbunden" sein sollen (Art. 4 g und 5 f., GCPRL). Für ihre Stellungnahme soll die zuständige Ethikkommission in diesen Fällen eine besondere Expertise auf dem Gebiet der Kinderheilkunde oder der betreffenden Krankheiten für Erwachsene vorweisen können oder sich entsprechend beraten lassen (Art. 4h und Art. 5 g, GCP-RL).

Die Ethikkommission ist auch für den Schutz der Probanden während der Prüfung zuständig. So soll sie über signifikante Änderungen am Prüfplan, die sich auf die Sicherheit der Prüfungsteilnehmer auswirken können, unterrichtet werden. 
Wenn sie diese Änderungen nicht gutheißt, darf der Sponsor den ursprünglichen Plan nicht ändern.

Auch weitere Bestimmungen sind für den Schutz der Probanden relevant, zum Beispiel die Kompetenz der Mitgliedstaaten, eine Prüfung zu stoppen (Art. 12, GCP-RL), oder die detaillierten Vorschriften zur Herstellung und Etikettierung der Prüfpräparate (Art. 13 GCP-RL und Art. 9-15 RL 2005). Die Bestimmungen zur Durchführung von Inspektionen oder zur Dokumentation stärken mittelbar den Schutz der Probanden. Ursprünglich sind sie für andere Zwecke definiert worden, die mit der Entwicklung des Arzneimittelbinnenmarktes eng verbunden sind.

\section{Die Stärkung des europäischen Arzneimittelbinnenmarkts}

Die Angleichung der Bestimmungen, die in den europäischen Mitgliedstaaten im Bereich der klinischen Prüfung anwendbar sind, steht im Zusammenhang mit der Vervollständigung des europäischen Arzneimittelbinnenmarktes (Erwägungsgrund 1, GCP-RL). Durch die Schaffung eines einheitlichen Kataloges wissenschaftlicher und ethischer Grundsätze für die klinische Prüfung soll die gegenseitige Anerkennung der für die Arzneimittelzulassung notwendigen Daten durch die zuständigen Behörden der Mitgliedstaaten und der Europäischen Union ermöglicht werden. Diese Verbesserungen dienen dem Arzneimittelbinnenmarkt, für dessen Verwirklichung Grundsätze, Kriterien und gemeinsame Verfahren für die Zulassung von Arzneimitteln in der EU seit 1965 schrittweise definiert worden sind. Nach der Analyse des Vorschlags für die GCP-RL durch den Berichterstatter (Amedeo 1998) sollen folgende Zwecke durch diese Richtlinie erreicht werden: 1) Die Erweiterung des Angebots und die Verbesserung der Qualität der Prüfungen, die durch die Änderung der Wettbewerbsbedingungen für Sponsoren und Forschungsinstitute erfolgen sollen. 2) Die wirtschaftliche Entwicklung der Industrie und die Zunahme der Investitionen der in dem Sektor der klinischen Prüfungen tätigen Unternehmen. 3) Der schnelle Zugang zu Innovationen für alle Patienten und insbesondere für Patienten mit schweren Erkrankungen, die im Rahmen einer klinischen Prüfung neue pharmakologische Substanzen vorzeitig bekommen können. Verschiedene Mechanismen, die zunächst die Phase vor der klinischen Prüfung betreffen, sind vorgesehen, um diese Verbesserungen zu erreichen. Einheitliche Regeln sollen den Sponsoren erlauben, in kurzer Zeit mit ihren klinischen Prüfungen zu beginnen (Art. 1 (1), GCP-RL). Die GCP-RL definiert ein System der impliziten Genehmigung, das dem Sponsor erlaubt, mit der klinischen Prüfung zu beginnen, wenn ein positives Votum der Ethikkommission vorliegt und die zuständige nationale Behörde keine Einwände erhoben hat. Die Ethikkommission sowie die zuständige nationale Behörde sollen in der Regel innerhalb einer maximalen Frist von 60 Tagen ihre Stellungnahme bzw. Einwände bekannt geben (Art. 6 (5) und 9 (4), GCP-RL). Für Prüfungen, die in mehreren Prüfungsorten auf dem Hoheitsgebiet eines Mitgliedsstaates stattfinden und den gleichen Prüfplan anwenden (sogenannte multizentrische klinische Prüfungen), wird nur die Stellungnahme einer einzigen Ethikkommission verlangt. Bei 
Prüfungen, die in mehreren Mitgliedstaaten durchgeführt werden, soll es pro Land eine einzige Stellungnahme geben (Art. 7 GCP-RL).

Weitere Mechanismen betreffen außerdem die Glaubwürdigkeit der bei klinischen Prüfungen erhobenen Daten, die durch Transparenz, Informationsaustausch und verschiedene Qualitätskontrollen gesichert werden soll. Transparenzanforderungen betreffen insbesondere Änderungen am Prüfplan (Art. 10 (a) GCP-RL), unerwartete schwerwiegende Nebenwirkungen (Art. 17 GCP-RL), sowie die Beendigung oder den Abbruch einer klinischen Prüfung (Art. 10 (c) GCP-RL). Signifikante Änderungen nach dem Beginn der klinischen Prüfung, die die Auslegung der wissenschaftlichen Dokumente, auf die die Durchführung der Prüfung gestützt ist, beeinflussen können, sollen bei der zuständigen Ethikkommission sowie der nationalen Behörde gemeldet bzw. von der Ethikkommission explizit und von der nationalen Behörde implizit befürwortet werden. Alle wichtigen Informationen über unerwartete schwerwiegende Nebenwirkungen sind schon im Verdachtsfall so rasch wie möglich den nationalen Behörden und Ethikkommissionen mitzuteilen und sollen in einem jährlichen Bericht an die Mitgliedstaaten aufgeführt werden (Art. 17 GCP-RL). Schließlich sollen auch die Gründe eines vorzeitigen Abbruchs der Prüfung diesen Institutionen mitgeteilt werden (Art. 10 c, GCP-RL).

Die Transparenz soll durch die Nutzung der für klinische Prüfungen mit Humanarzneimitteln in Europa hergestellten Datenbank (Krafft 2005) gefördert werden. Informationen zu Genehmigungsanträgen, Stellungnahmen der Ethikkommission, Änderungen des Prüfplans, unerwünschten Ereignissen (Art. 17 abs. 3 (a) GCPRL) und Abschluss der klinischen Prüfungen müssen durch diese Datenbank für alle zuständigen nationalen Behörden, die europäische Arzneimittelagentur und die europäische Kommission zugänglich sein (Art. 11 (1) GCP-RL). Genauso müssen die durchgeführten Inspektionen zur Überwachung von Prüfärzten, Prüfzentren und Sponsoren bezüglich Konformität mit der guten klinischen Praxis in der Datenbank vermerkt sein (Sewing 2011). Diese Inspektionen sind nicht mit der laufenden unabhängigen Kontrolle zu verwechseln, die im Auftrag des Sponsors zur Überprüfung der Qualität der Studiendurchführung und der Studiendaten durchgeführt werden muss (Art. 16, 2005-RL). Sie werden durch die nationalen Arzneimittelbehörden im Namen der Europäischen Gemeinschaft (Art. 15, GCP-RL) und gemäß den in der 2005-RL festgelegten Bestimmungen zu den Unterlagen, die vom Sponsor archiviert werden müssen (Art. 16-20, 2005-RL), zu den Inspektionsverfahren (Art. 23-30, 2005-RL) und zur Ernennung der Inspektoren (Art. 21, 22, 2005-RL) durchgeführt.

\section{Der europäische Reformvorschlag}

Die Definition neuer Anforderungen für die Durchführung von klinischen Prüfungen mittels Richtlinien ist auf viel Kritik gestoßen (Heidenreich 2005). Diese Kritik findet sich zum Teil in der Begründung der europäischen Kommission zu ihrem Vorschlag vom 17. Juli. 2012 für eine Verordnung über klinische Prüfungen mit Hu- 
manarzneimitteln (Europäischen Kommission 2012a) wieder, die die GCP-Richtlinie sowie die 2005-RL ersetzen soll (Stand 2013). Nach Ansicht der Kommission sind die Folgen der oben beschriebenen Mechanismen im Hinblick auf das Ziel der Förderung der Industrie eher negativ ausgefallen. Ein hohes Schutzniveau für Prüfungsteilnehmende ist zwar erreicht worden, aber die GCP-Richtlinie hat auch „direkte Auswirkungen auf die Kosten und die Durchführbarkeit klinischer Prüfungen gehabt, die ihrerseits dazu geführt haben, dass die Aktivität im Bereich klinischer Prüfungen in der EU nachgelassen hat". Laut Kommission ist die Zahl der Anträge auf Arzneimittelstudien in der Europäischen Union von 2007 bis 2011 um $25 \%$ zurückgegangen. Die Wettbewerbsfähigkeit der europäischen Union in diesem Bereich sei dementsprechend unzureichend und wirke sich negativ auf die Kapazität von Firmen sowie nicht kommerziellen Forschungsinstitutionen aus, neue und innovative Arzneimittel zu entwickeln. Mehrere Bestimmungen der Richtlinie 2001/20/EG sind in den Begleitunterlagen zum Vorschlag als Ursache hierfür identifiziert worden. Dazu gehören zum Beispiel die rechtlichen Maßnahmen, die Verzögerungen vor Beginn klinischer Prüfungen zur Folge haben können: die separaten Bewertungsverfahren der Anträge durch jeden betroffenen Mitgliedstaat sowie die Beteiligung mehrerer nationaler Behörden oder Ethikkommissionen an der Bewertung der Anträge (Europäische Kommission 2012b).

Das Hauptziel des Kommissionsvorschlags (Begründung, 3.14) ist durch eine effizientere Harmonisierung der für klinische Forschung anwendbaren Bestimmungen den freien Arzneimittelverkehrs in der EU zu vervollständigen. Dabei muss der Patientenschutz gewährleistet bleiben, aber die Balance zwischen den beiden Zielen scheint hier eine andere als in der GCP-RL zu sein. Besonders bemerkenswert in dieser Hinsicht sind folgende neue Bestimmungen zur Bewertung der Anträge auf Genehmigung einer klinischen Prüfung: Verkürzung der Bewertungsfristen (Art. 6 und 8), Trennung zwischen wissenschaftlicher und ethischer Bewertung der Prüfungsvertretbarkeit (Art. 5 und 7), und schließlich der faktische Ausschluss lokaler Ethikkommissionen vom Zulassungsverfahren für klinische Prüfungen. Dabei bleibt es den Mitgliedstaaten überlassen, welche internen Institutionen die ethische Bewertung durchführen sollen (Art. 9 und 10).

Dieser Verordnungsvorschlag ist schon auf starke Kritik in Deutschland gestoßen, insbesondere weil die neuen Regelungen ,,mit international anerkannten ethischen Standards brechen (...) und die Sicherheit der Studienteilnehmer in nicht vertretbarer Weise erheblich gefährde[n]" (Arbeitskreis Medizinischer Ethikkommissionen 2012). Die Sorge um die Beachtung international anerkannter ethischer Standards und um die Sicherheit der Studienteilnehmer wird von den beratenden Institutionen der Europäischen Organe geteilt. So hat der Europäische Wirtschaftsund Sozialausschuss (EWSA) in seiner Stellungnahme zum Kommissionsvorschlag den europäischen Gesetzgeber aufgerufen, nur klinische Prüfungen zuzulassen, die - in Einklang mit den Anforderungen der Helsinki Deklaration - die positive Stellungnahme einer unabhängigen Ethikkommission erhalten haben (EWSA 2012). Der Ausschuss für Umweltfragen, öffentliche Gesundheit und Lebensmittelsicherheit des europäischen Parlaments (europäisches Parlament 2013) geht in seinem Bericht über den Vorschlag der Kommission noch weiter. In zahlreichen Ände- 
rungsanträgen fordert der Ausschuss insbesondere die Beteiligung einer Ethikkommission beim Genehmigungsverfahren für neue klinische Prüfungen, den verstärkten Schutz des einzelnen Probanden durch weitere Vorkehrungen, die Vertiefung der Zusammenarbeit der nationalen Ethikkommissionen und die Transparenz der durch klinische Prüfungen gewonnenen Daten. Schließlich wurden auch zusätzliche Schutzmaßnahmen für besonders schutzbedürftige Probanden gefordert (Europäisches Parlament 2013).

Die Diskussionen um den Vorschlag sollen in den kommenden Monaten weitergeführt werden. Die neue Rechtsverordnung soll ab 2016 anwendbar sein.

\section{Schrifttum}

Amedeo A (1998) Stellungnahme. http://www.europarl.europa.eu/sides/getDoc.do?pubRef=-// $\mathrm{EP} / / \mathrm{TEXT}+\mathrm{REPORT}+\mathrm{A} 4-1998-0407+0+\mathrm{DOC}+\mathrm{XML}+\mathrm{V} 0 / / \mathrm{DE} \#$ Contentd3477100e1229. Zugegriffen: 21. Mai 2013

Arbeitskreis Medizinischer Ethikkommissionen (2012) Stellungnahme. http://www.ak-med-ethik-komm.de/documents/StellungnahmeEUVerordnungklinischePruefungen.pdf. Zugegriffen: 21. Mai 2013

EU Kommission (2012a) Vorschlag für eine Verordnung des europäischen Parlaments und des Rates über klinische Prüfungen mit Humanarzneimitteln und zur Aufhebung der Richtlinie 2001/20/EG (COM (2012) 369 final). http://eur-lex.europa.eu/LexUriServ/LexUriServ.do?uri $=$ COM:2012:0369:FIN:DE:PDF. Zugegriffen: 21. Mai 2013

EU Kommission (2012b) Arbeitsunterlagen der Kommissionsdienststellen, Begleitunterlage zum Vorschlag für eine Verordnung (SWD (2012) 200 final). http://ec.europa.eu/health/files/clinicaltrials/2012_07/summary/2012_07_summary_de.pdf. Zugegriffen: 21. Mai 2013

Europäisches Parlament, Ausschuss für Umweltfragen, öffentliche Gesundheit und Lebensmittelsicherheit (2013) Entwurf eines Berichts über den Vorschlag für eine Verordnung über klinische Prüfungen. http://www.europarl.europa.eu/sides/getDoc.do?pubRef=-//EP// NONSGML+COMPARL+PE-504.236+01+DOC+PDF+V0//DE\&language=DE. Zugegriffen: 21. Mai 2013

EWSA (2012) Stellungnahme INT/658 klinische Prüfungen mit Humanarzneimitteln. http://eescopinions.eesc.europa.eu/eescopiniondocument.aspx?language $=$ de $\&$ docnr $=2059 \&$ year $=2012$. Zugegriffen: 21. Mai 2013

Heidenreich K, Möritz A, Löffler H, Oberle-Rolle B (2005) Klinische Prüfungen in Deutschland und der EU im neuen gesetzlichen Umfeld. Bundesgesundheitsblatt 48:415-422

Krafft H (2005) Die Eudra CT-Datenbank bei der EMEA zur Erfassung klinischer Prüfungen in Europa. Bundesgesundheitsblatt 48:453-458

Sewing K-F (2011) Die GCP-Richtlinie: Balance zwischen Sicherheit und Bürokratie? In: Deutsch E, Duttge G, Schreiber H-L, Spickhoff A, Taupitz J (Hrsg) Die Implementierung der GCPRichtlinie und ihre Ausstrahlungswirkungen. Springer, Heidelberg, S 63-68 